\title{
Low Radar Cross-section and Low Cost Dipole Antenna Reflector
}

\author{
G. G. Machado ${ }^{1}$, M. T. de Melo ${ }^{1}$, H. V. H. Silva Filho ${ }^{1}$ \\ A. G. Neto ${ }^{2}$, T. R. de Souza ${ }^{2}$ \\ ${ }^{1}$ Department of Electronics and Systems, Federal University of Pernambuco, Recife, Brazil \\ ${ }^{2}$ Federal Institute of Paraíba, João Pessoa, Brazil \\ *corresponding author, E-mail: gabriel.gm91@gmail.com
}

\begin{abstract}
This paper presents a method for reducing Radar CrossSection (RCS) of an increased gain metal backed dipole antenna. Numerical simulations were done and compared to a laboratory experiment. The results show that when a Perfect Electrical Conductor (PEC) is replaced by a Frequency Selective Surface (FSS), the antenna is still able to perform with the desired characteristics, but the RCS of the structure is greatly reduced out of band. The design of the FSS and the return loss, gain improvement, and RCS are presented for an antenna operating at $4.2 \mathrm{GHz}$, and the results are compared with a conventional metal backed layout. Measurements show a good agreement with the simulations, and so the advantages on other structures from the reviewed literature are mentioned.
\end{abstract}

\section{Introduction}

Dipole antennas have been used for its simplicity and capability of radiating signals in an omnidirectional pattern. They are used specially for mobile communications [1]. On the other hand, the disadvantage of using this kind of antenna is due its low gain, which does not exceed 2.5dBi [2].

In this manner, a way of increasing a half-wavelength dipole antenna, is placing a metal plate at a correct distance from it [1]. This conducting background will reflect the signal, changing the antenna's radiation pattern, which reflects in greater directivity and gain. This configuration of metal backed antenna also increases the Radar Cross-section (RCS) of the structure, representing a drawback for military applications. Moreover, other works were published on metamaterial backed dipole antennas for high gain [3]. At this report, no study about the RCS has been done, although metamaterials are showing a good performance for RCS reduction $[4,5]$.

As the demand for the stealth budged in warfare increases, researches have been published in the open literature which shows that radar backscatter suppression can be achieved using thin carbon based microwave absorbers and resistive metamaterials [6-9]. Frequency selective surfaces are mainly used as microwave filters, for applications such as frequency separation in satellites [10]. Furthermore, FSS can also be used as a reflective background to antennas, for beam direction switching purposes $[11,12]$.

Preview works with FSS and antennas were reported in [13], aiming to enhance the directivity of a microstrip antenna. In this kind of work, the FSS is used as a resonator, and acts like a partial reflective surface [14]. In [15] a great RCS reduction was reported within the antenna's band, but results for the reflection coefficient show that out of band the RCS increases with frequency . Additionally, Turpin [16] reported a good RCS reduction within 1-12 $\mathrm{GHz}$, for an antenna based on absorbing materials. The design consists of a planar antenna over a FSS and absorbing material. Although the results were consistent with today's demand for RCS, the costs for using these techniques are higher due to the materials used in this process. Also, H.$\mathrm{Y} ., \mathrm{Xu}$ [17] reported a monopole antenna design with a low RCS for Ultra-Wideband (UWB) applications. The design favors a UWB for the antenna, keeping a low RCS. But results show that the Radar Cross-section increases with frequency, which is one of the concerns of this paper.

Furthermore, literature shows techniques to reduce RCS for antenna's reflectors applications [18-25]. In [26], Y. Liu reports a low RCS microstrip antenna, replacing a solid metal background by a FSS.

This work contributes with a lower cost project than the reported, it shows details for this technique using a square loop FSS in order to reflect the signal from a linear wire half-wavelength dipole antenna. This is done by comparing theoretical models, simulations, performed in a full EM simulator, with measured results in laboratory. Moreover, the low cost is achieved by using a FSS screen over a FR4 dielectric, which is a known low cost substrate for RF applications. In addition, there is no use of radar absorbing materials, or any other kind of substrates that can be translated into higher cost for the project. Furthermore, the results show that the antenna performance is not reduced in exchange for RCS reduction. Also, it can be noticed that, out of the frequency of interest, the RCS of the entire system keeps decreasing, while frequency increases. On the other hand, the referenced works show a good RCS reducion in band, but the radar cross-section increases for frequencies out of band. That means that, for radar systems operating frequencies above the UWB reported by those works, the reflection coefficient becomes significantly high out of band.

The replacement of the metal background for a FSS results in a significant RCS reduction for frequencies outside its operation band $[24,26]$. This happens because the FSS 


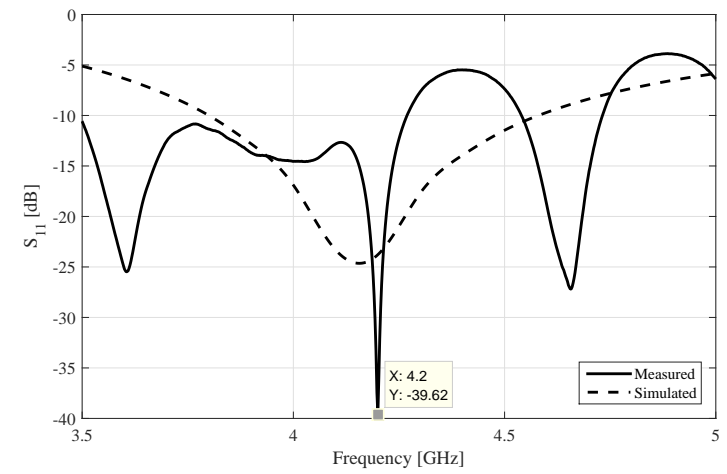

Figure 1: Comparison between the simulated and measured antenna

will reflect only the signals at the antenna's frequency band, being transparent to radar signals out of this band. The intention of this work is to keep a low RCS out of band. It is expected that the radar back-scatter for frequencies outside the antenna's and FSS' bandwidth to be reduced as close to zero as possible. Furthermore, the antenna's performance should not be affected by this replacement, i.e., the gain and the impedance matching must remain within an acceptable difference from the antenna and metal background configuration.

\section{Methodology}

For this feasibility study, a half wavelength linear dipole antenna was chosen because of its simplicity and well known radiation patterns [1]. The antenna's gap and wire radius were defined by the available coaxial cable, thus, those parameters were inserted for the simulations. For the available coaxial cable, the conductor's radius was $0.5 \mathrm{~mm}$ and the feeding gap was $3 \mathrm{~mm}$.

As shown by the frequency allocation chart in [27], one of the aeronautical navigation bands is within $4.2-4.4 \mathrm{GHz}$. As consequence of this, the chosen frequency for the antenna was $4.2 \mathrm{GHz}$. Thus, from that definition, the theoretical length of the antenna is: $l=\frac{\lambda}{2}=35.7 \mathrm{~mm}$. The actual size of the antenna was $36.3 \mathrm{~mm}$, being approximately $1.7 \%$ larger than the theoretical length. From these simple calculations, the antenna was designed in CST Microwave Studio, using open add space boundary conditions, and later the dipole antenna was fabricated. The results for $S_{11}$ are shown in Fig. 1.

It can be noted that the antenna has a good resonance at $4.2 \mathrm{GHz}$, and the measured dipole had actually a better performance at the desired frequency than the simulated. As the wire width and gap could not be altered due to the cable used, a better control of the antenna's bandwidth was not possible to obtain.

Other resonant frequencies were observed for the antenna at approximately $3.6 \mathrm{GHz}$ and $4.6 \mathrm{GHz}$. They are probably a result of the antenna's fabrication process, where a simple coaxial cable was used to build it. Those frequen- cies, as it will be shown in the next section, will not be reflected by the FSS, thus they should not interfere in later measurements.

Additionally, by comparison to the aforementioned works, this antenna has a very narrow band to accomplish a low RCS signature for frequencies out of band.

\subsection{FSS Design}

This approach for RCS reduction was chosen by the capability of a FSS to perform as a PEC in band, and as a transparent surface out of band. Thus, for having low reflections out of band, the RCS is greatly reduced, as it is a function of the reflected power of the radar over the surface. This will be later demonstrated by simulations and laboratory measurements.

In this manner, the chosen structure for the FSS was a square loop design. This design was chosen due to its in band performance and frequency stability [28, 29]. A square loop FSS and its equivalent circuit are shown in Fig. 2 [30-32].

From Fig. 2, the equivalent inductive reactance $\left(X_{L}\right)$ is given by (1) [31]:

$$
\frac{X_{L}}{Z_{0}}=\frac{d}{p} F(p, 2 w, \lambda, \phi),
$$

and the capacitive susceptance is given by (2):

$$
\frac{B_{C}}{Z_{0}}=2 \epsilon_{r} \frac{d}{p} F(p, g, \lambda, \theta)
$$

The function $F$ is given by (3):

$$
\begin{aligned}
F(p, w, \lambda, \phi)= & \frac{p \cos \phi}{\lambda}\left\{\ln \left[\operatorname{cossec}\left(\frac{\pi w}{2 p}\right)\right]+\right. \\
& +G(p, w \lambda, \phi)\}
\end{aligned}
$$

The second auxiliary function $G$ is given by (4)

$G(p, w \lambda, \phi)=$

$$
\frac{0,5\left(1-\beta_{2}\right)^{2}\left[\left(1 \frac{\beta^{2}}{4}\right) C_{+}+C_{-}+4 \beta^{2} C_{+} C_{-}\right]}{\left(1 \frac{\beta^{2}}{4}\right)+\beta^{2}\left(1+\frac{\beta^{2}}{2}-\frac{\beta^{4}}{8}\right) C_{+}-C_{-}+2 \beta^{6} C_{+} C_{-}}
$$

Finally, $\mathrm{C}$ and $\beta$ can be calculated by:

$$
\begin{gathered}
C_{ \pm}=\frac{1}{\sqrt{1 \pm \frac{2 p \sin \phi}{\lambda}-\left(\frac{p \cos \phi}{\lambda}\right)^{2}}}-1 \\
\beta=\sin \left(\frac{\pi w}{2 p}\right)
\end{gathered}
$$

In which $\theta$ and $\phi$ are angle of incidence. Then, the transmission coefficient can be calculated by (7):

$$
T=\frac{1}{X_{L}-\frac{1}{B_{C}}}
$$




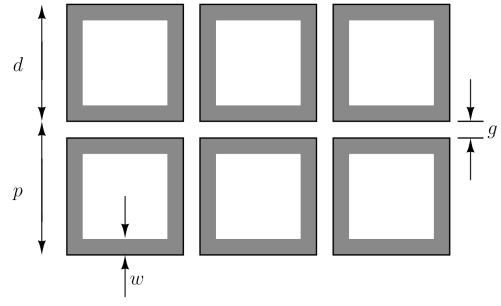

(a)

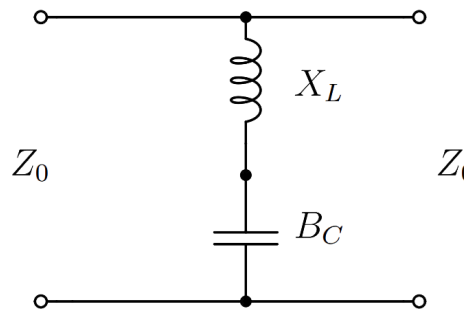

(b)

Figure 2: Square loop strucure (a); equivalent circuit (b)

Then the transmitted power, $P_{T}$, is given by [32]:

$$
P_{T}=\frac{1}{1+0.25 T^{2}}
$$

The FSS was to be printed on FR4 substrate, which $\epsilon_{r}=4.4, h=1.6 \mathrm{~mm}$ and $\tan \delta=0.02$. The FR4 was chosen for being a cheap substrate, which showed a good performance for the FSS. The final dimensions for the FSS were as described:

- $d=17.8 \mathrm{~mm}$;

- $p=24 m m$;

- $w=1.5 \mathrm{~mm}$

- $g=6.2 \mathrm{~mm}$;

- FSS total lateral size $=21.6 \mathrm{~cm}$;

- FSS full structure area $=466.56 \mathrm{~cm}^{2}$

Having these FSS parameters, 1 and 2 can be used to calculate the inductive reactance $\left(X_{L}\right)$ and capacitive susceptance $\left(B_{C}\right)$. Thus, a code was developed in Matlab based on [33] to obtain the power transmission curve in $\mathrm{dB}$ based on the values of these parameters, which were calculated as $X_{L}=0.21 \Omega$ and $B_{c}=10.24 \Omega^{-1}$. So, using 9 and 10 the capacitance and inductance values were calculated. Their respective values were found to be $\mathrm{C}=3.70 \mathrm{pF}$ and $\mathrm{L}=0.38 \mathrm{mH}$.

Moreover, CST Design was used, to compare the simulated circuit to the numerical method on Matlab. Fig.3 presents a simplified flow chart representing the routines of the code used for calculating parameters.

$$
C=\frac{1}{2 \pi f_{c} B_{c}}
$$

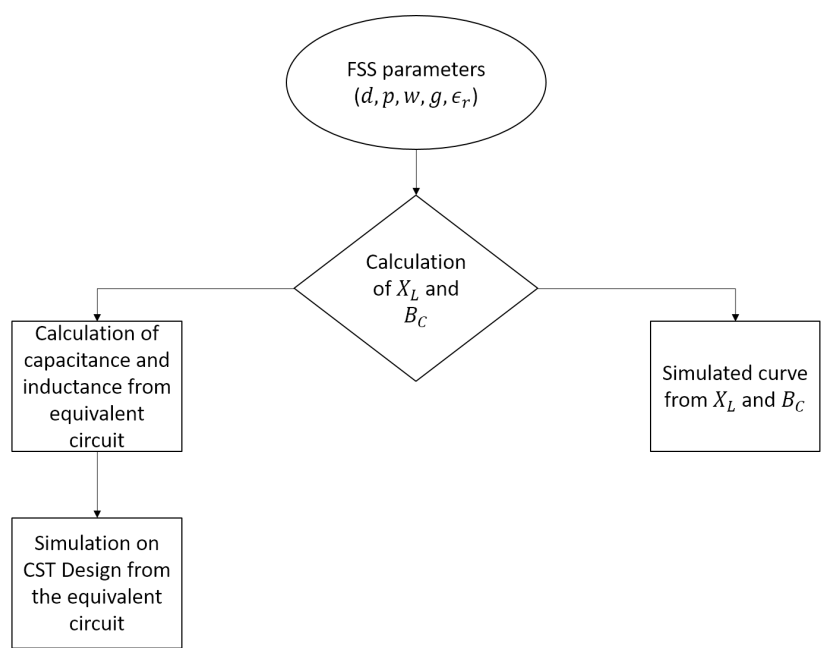

Figure 3: Methodology for the theoretical model simulation

$$
L=\frac{1}{\left(2 \pi f_{c}\right)^{2} C}
$$

Additionally, simulations were done using the unity cell boundary condition on CST Microwave Studio, with Floquet ports exciting plane waves at TE and TM polarization modes. Also, a simulation of the complete FSS structure with the antennas was also done to validate the unity cell calculation. This simulation was performed using the open add space boundary condition to allow the radiated signal to propagate through the FSS with no reflections from the boundaries. As for the RCS simulation, PML boundary condition was used with the Integral Equation Solver, for time optimization. Fig. 4 shows the results for the unity cell, complete structure, and also for the theoretic model.

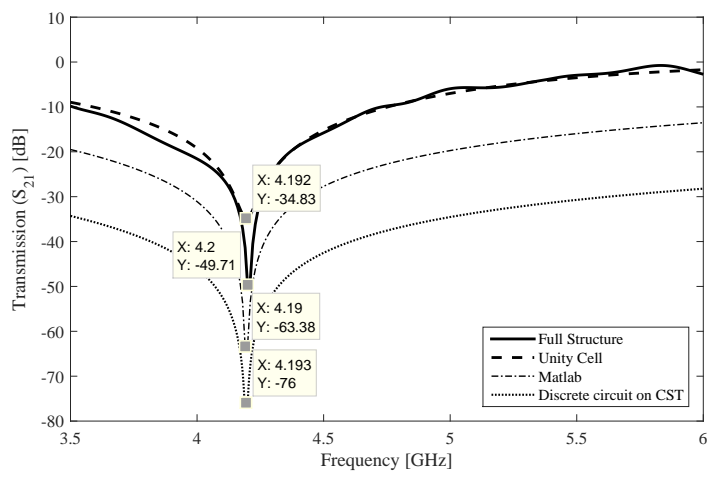

Figure 4: Comparison between the simulated results for unity cell, theoretical model, and the complete structure

It can be seen that both unity cell and full structure results agree, with its minimum at the desired frequency of $4.2 \mathrm{GHz}$. In addition, Table 1 the relative error in percent of the resonance frequencies theoretical models in relation to the measurement, whose frequency was $4.2 \mathrm{GHz}$. 
Table 1: Relative error from simulated and theoretical models

\begin{tabular}{cc}
\hline Models & Relative error \% \\
\hline Unity Cell & 0.19 \\
Complete Structure & 0 \\
Matlab & 0.23 \\
Discrete Circuit & 0.16 \\
\hline
\end{tabular}

\subsubsection{FSS as a reflective background}

As the FSS had its rejection band at the same range as the antenna, simulations were done to compare the performance of the designed FSS as a reflective background, to a metal plate having the same area.

A dipole antenna will reflect its signal, with a good impedance matching with the ground plane, when it is carefully placed at a distance of $\lambda / 4$ above the conductive surface [1].

Fig. 5 (a) and (b) show the results for both systems. As it can be seen, the directivity of the antenna was increased for both configurations. These results show that the replacement of the metal plate by the FSS does not implies on a significant decrease in gain.

The 90 degrees rotation which can be seen between Fig. 5 (a) and (b) happens inside the simulation software, CST Microwave Studio. The results depicted on Fig. 5, had a gain of $8.34 \mathrm{dBi}$ for the antenna-metal plate configuration, and $7.89 \mathrm{dBi}$ for the antenna-FSS configuration. While the gain for the dipole antenna in free-space is $2 \mathrm{dBi}$, those results represent a gain of $5.89 \mathrm{dBi}$, or 3.9 times more power, for the antenna-FSS scenario.

\section{Measurements and discussion}

\subsection{FSS measurement}

For this experiment, a reference level for the FSS was measured to level the maximum transmission to $0 \mathrm{~dB}$. For that, two Pasternak WR187 horn antennas were used, having its operating bandwidth between 3.95 and $5.85 \mathrm{GHz}$. This antenna has a minimum gain of $20 \mathrm{dBi}$. In addition, The Agilent E5071C VNA was used for all the measurements. The set-up can be seen in Fig. 6, and this exact situation was also simulated (Fig. 4).

The transmission measured in laboratory matched the expected results from simulation, as seen in Fig. 7:

As the reflections are expected to be higher at $4.2 \mathrm{GHz}$, and almost no signal reflected outside the FSS bandwidth, it is expected that the antenna will be able to reflect its signal, increasing the gain. This is described in the next section.

The reflection coefficient from Fig. 7 shows to be at least $20 \mathrm{~dB}$ higher than previous works [6,9], and also having a very narrow band of rejection. Moreover, this characteristic will contribute in the RCS reduction out of the antenna's band, as there will be very low reflections for frequencies higher than $4.2 \mathrm{GHz}$.

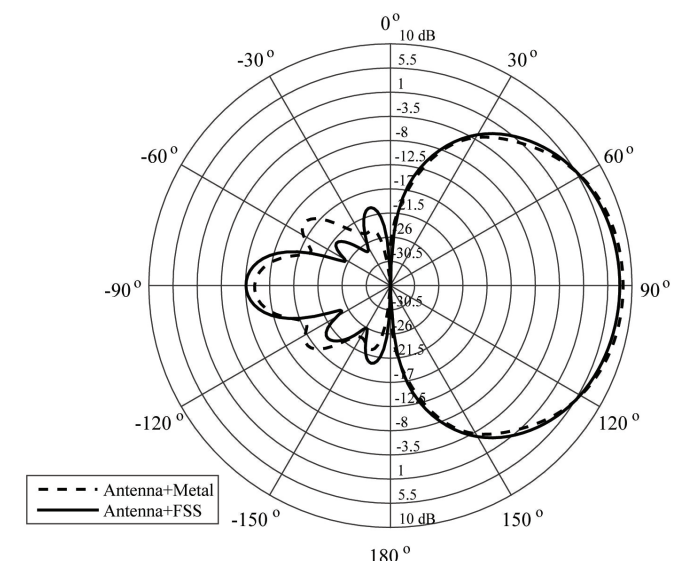

(a)

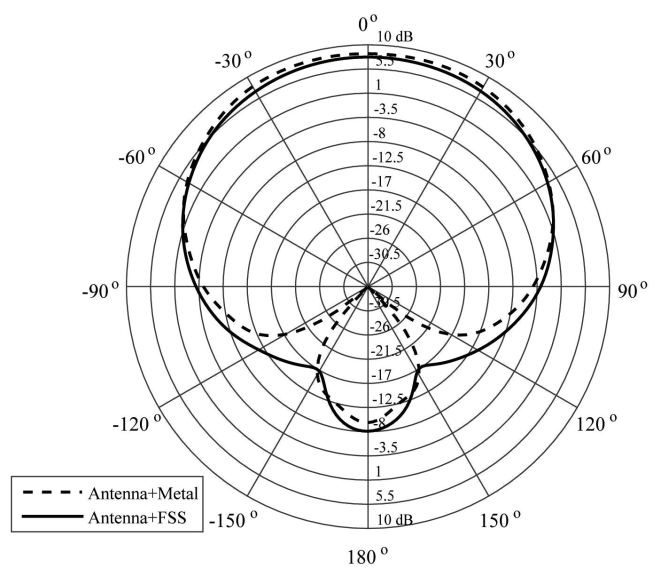

(b)

Figure 5: Radiation pattern for the antenna on both configurations at $\phi=0$ (a), and $\theta=90$ (b)

\subsection{Antenna performance measurements}

The first step for the antenna measurement was regarding the impedance matching with the FSS. The antenna was placed at a quarter wavelength far from the FSS, and the $S_{11}$ was then measured. A picture from the measurement can be seen in Fig. 8(a), while the result is shown in Fig. 8(b).

It is noted that a small frequency shift of $30 \mathrm{MHz}$ occurred during the measurements. Also, a small oscillation happens for the measured $S_{11}$. This may happen due to standing waves which causes constructive and destructive interference during this process. This could be reduced if the experiment could be made in a proper anechoic chamber, but such facility is not available at the universities.

As there was not a place available to make a proper radiation pattern measurement for the antenna, 5 scenarios 


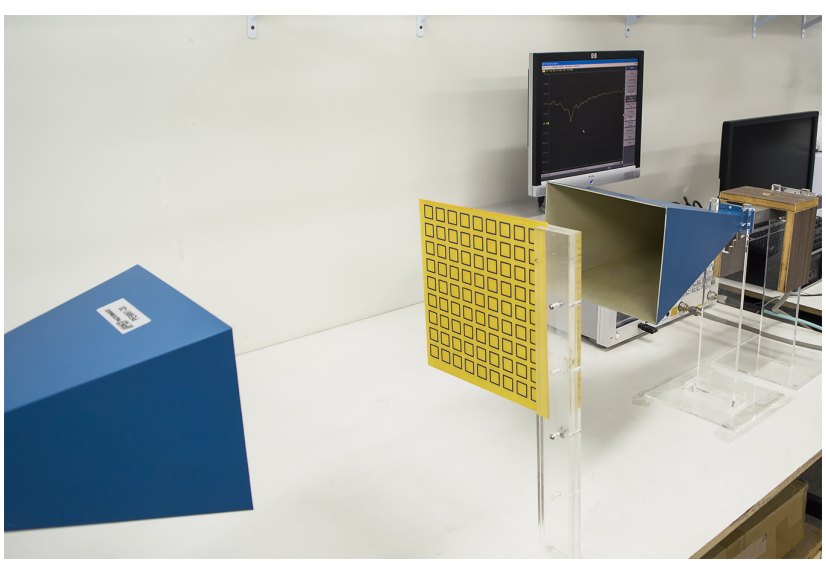

Figure 6: Setup used for the FSS transmission measurement.

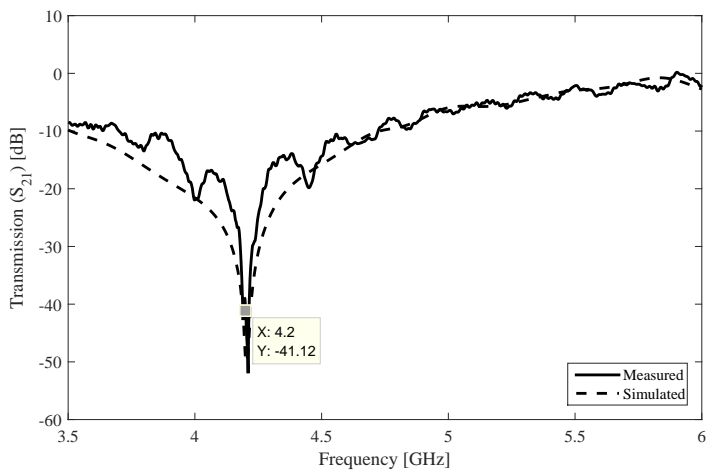

Figure 7: Comparison between measured and simulated transmission for the FSS.

were assembled, trying to overcome this problem. These are described in Fig. 9:

The idea for that, is to verify whether there is actually more signal being received by the horn antenna, when a reflective background is placed.

The results for those measurements are shown in Figs. 10(a) and 10(b).

It can be seen that, even without the complete radiation pattern (which was not possible to obtain, due to lack of laboratory resources), the FSS was capable of reflecting the signal from the antenna, almost with the same intensity as the metal plate, with only $0.9 \mathrm{~dB}$ of difference.

These results show that the FSS replacing a metal background does not degrade the antenna's performance. This could also be seen at the simulated results. As the preview measured results agreed with the predicted in the simulation, it is safe to affirm that the antenna's radiation performance did not change with the proposed replacement.

\subsection{RCS Measurements}

The bistatic RCS $\left(\sigma_{t g t}\right)$ measurements were done using the same Agilent E5071C VNA, at the frequency domain. The FSS and the metal plate were placed at $70 \mathrm{~cm}$ from the horn

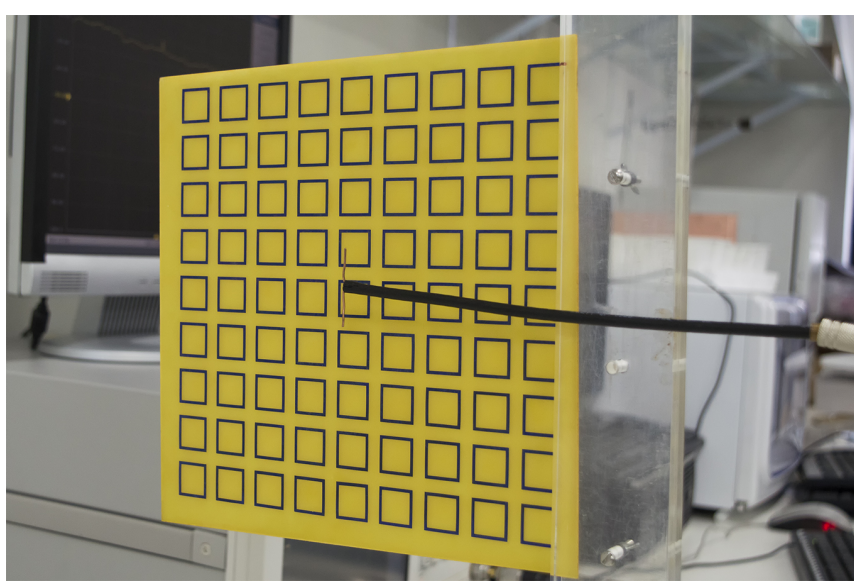

(a)

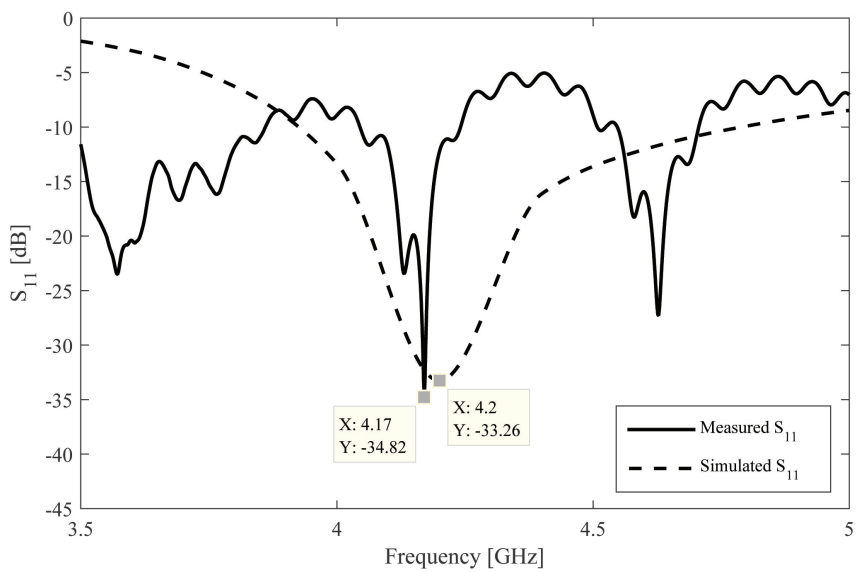

(b)

Figure 8: (a) Measurement Process; (b) Results for the simulated and measured experiment with the FSS as a reflective background.

antennas, and the received power was measured. The obtained points (Fig. 11(a)) were later put into Eq. 11, to obtain the RCS for the FSS and the the metal plate (both having the exact same area). The parameters on Eq. 11 represented by $G_{t}$ and $G_{r}$ are the antennas' gain, while $\lambda$ is the wavelength, $R$ is the distance between the antenna and the target, $P_{r}$ and $P_{t}$ are the received and transmitted power, respectively $\left(P_{r}=10^{\frac{S_{21}}{10}}\right)$.

$$
\sigma_{t g t}=\frac{P_{r}}{P_{t}}=\left[\frac{(4 \pi)^{3} R^{4}}{G_{t} G_{r} \lambda^{2}}\right]
$$

Fig. 11(b) shows the measured RCS from the FSS and the Metal plate. While Figure 12(b) shows the comparison between the measured and predicted results from the EM simulator.

From the experimental results, the RCS for the FSS and the metal plate clearly follows the predicted behaviour, as seen on Fig. 12(a) and Fig 12(b). While a very low RCS was measured out of the FSS operating band, an RCS equivalent to the metal plate was shown for frequencies in band. 
$\square$

b)

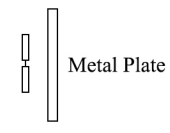

Horn Antenna

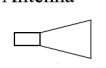

a)

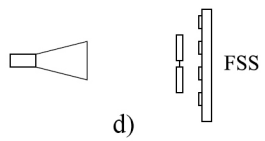

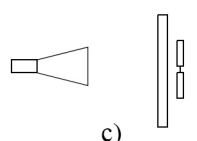
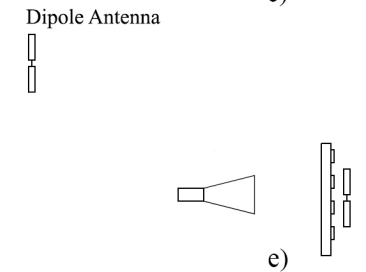

Figure 9: Scenarios for the antenna measurement. a) Free space; b) Antenna and metal plate reflecting; c) Antenna and metal plate blocking; d) Antenna and FSS reflecting; e) Antenna and FSS Blocking.

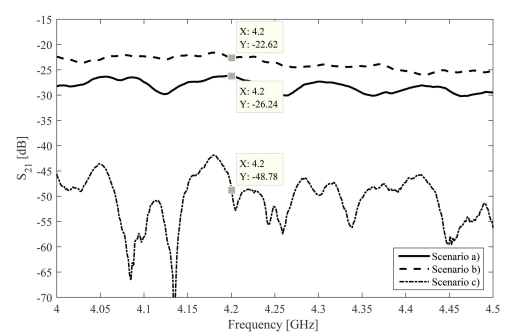

(a)

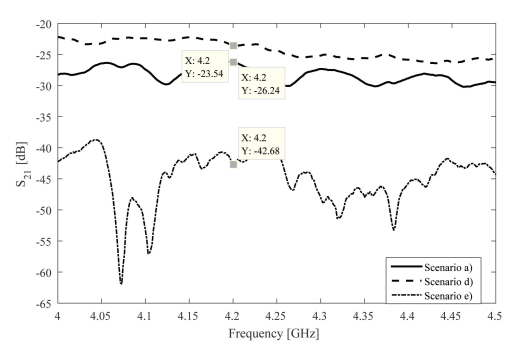

(b)

Figure 10: (a) Measurements for the metal plate configuration; (b) Measurements for the FSS configuration

These results, depicted by Fig. 11(b), contributes for a reduction on the out-of-band radar backscatter, while the the gain of a dipole antenna is increased, when both antenna and FSS operate at the same frequency range. It is also important to note that a wider band could not be measured, as the available antennas had a frequency range from 3.5-

\section{$5.85 \mathrm{GHz}$.}

When compared to the work of UWB Antennas with low RCS $[6,17,19]$, the results presented on this paper shows the advantage of operating in a single and narrow frequency for stealth systems. While for UWB antennas the RCS increases with the frequency, operating on a narrow band shows that the radar cross-section is only significant for the antenna's frequency band, and it tends to zero for higher frequencies.

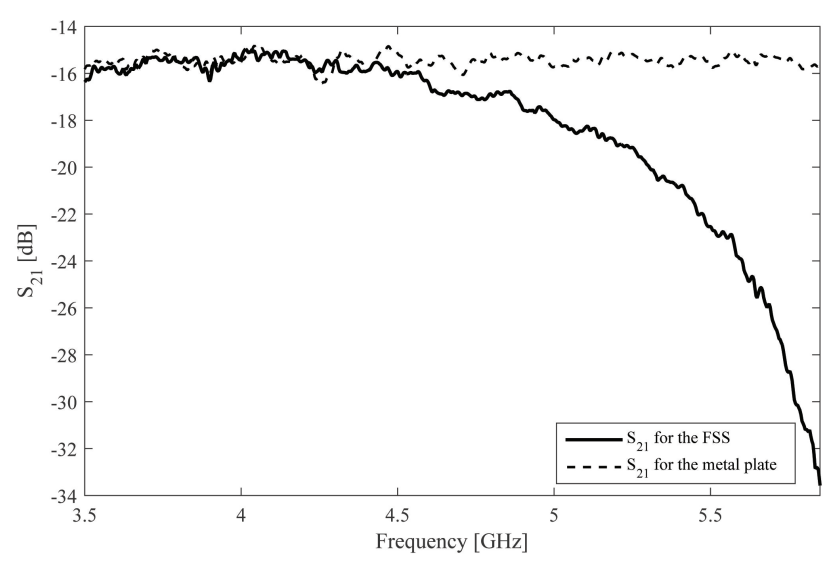

(a)

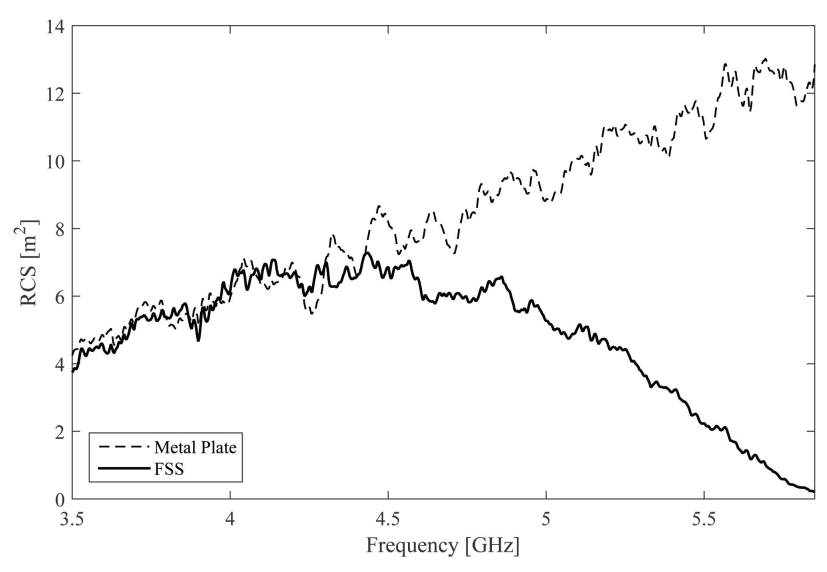

(b)

Figure 11: (a) Measured $S_{21}$ for the FSS and Metal Plate; (b)Measured RCS for the FSS and metal plate.

\section{Conclusion}

This paper presented the simulations and measurements for a square loop FSS, comparing its performance with a reflecting metal plane and discussing the results.

Simulations showed the construction of a layout capable of increasing a dipole antenna gain, replacing a traditional conducting surface by the designed FSS. This arrangement was compared to the metal background, to verify the performance of both systems. The results showed that both configurations perform similarly, with no major decrease in gain for the FSS as an antenna reflector. A theoretical model for the FSS was developed to compare the simulations to the literature review for this kind of FSS. Furthermore, the results from the mathematical model were kept within a $0.23 \%$ of error compared to the measured results.

RCS simulations and measurements were described and compared, showing a sensitive decrease in the RCS of the whole system, when the metal plate was replaced by the FSS. This work contributed with theoretical and practical results, showing a novel method to achieve a better gain for a dipole, keeping its RCS low for frequencies out of band. 


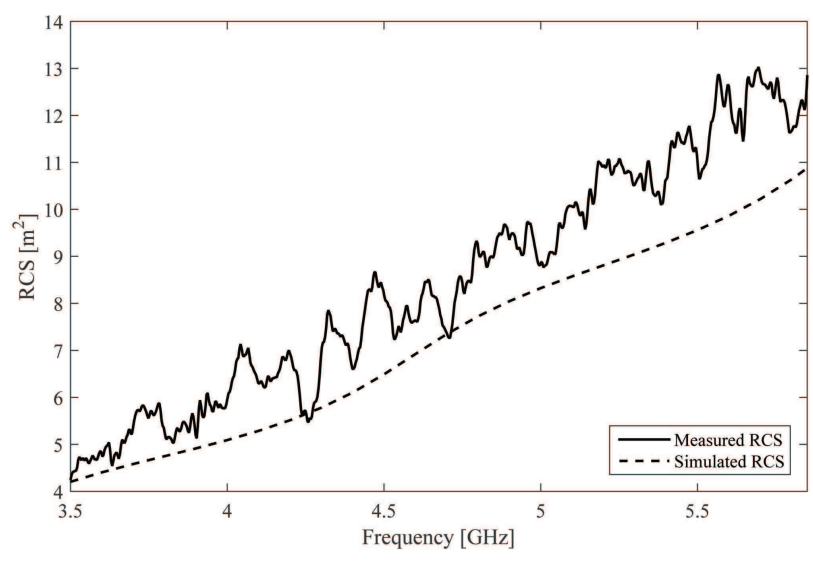

(a)

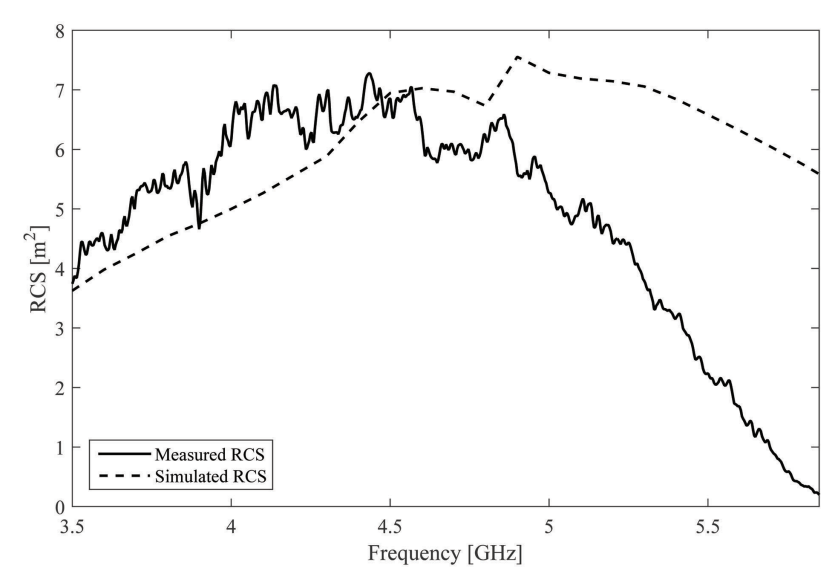

(b)

Figure 12: (a) Comparison between simulation and measurement for the metal plate; (b)Comparison between simulation and measurement for the FSS.

\section{Acknowledgement}

This work has been supported by the National Counsel of Technological and Scientific Development (CNPq) from Brazil, during overseas studies in Queen's University Belfast. I also would like to thank Professor Robert Cahill, for assisting in the beginning of this work.

\section{References}

[1] C. A. Balanis, Antenna Theory: Analysis and Design. Wiley, 2012.

[2] W. L. Stutzman and G. Thiele, Antenna theory and design. J. Wiley, 1998.

[3] V. Sarin, M. Jayakrishnan, C. Aanandan, M. Pezholil, and V. Kesavath, "A metamaterial backed dipole antenna for high gain directional communications," Advanced Electromagnetics, vol. 5, no. 1, pp. 9-14, 2016.

[4] F. Costa and A. Monorchio, "Electromagnetic absorbers based on high-impedance surfaces: From ultra-narrowband to ultra-wideband absorption," $A d$ vanced Electromagnetics, vol. 1, no. 3, pp. 7-12, 2012.

[5] M. A. Abdalla and Z. Hu, "On the study of development of x band metamaterial radar absorber," $A d$ vanced Electromagnetics, vol. 1, no. 3, pp. 94-98, 2012.

[6] Y. Han and Z. Xue, "A ultrathin and wideband radar absorber using slotted treble-square-loops resistive fss," in Environmental Electromagnetics (CEEM), 2015 7th Asia-Pacific Conference on, Nov 2015, pp. 27-30.

[7] F. Costa, A. Monorchio, and G. Manara, "Ultra-thin absorbers by using high impedance surfaces with resistive frequency selective surfaces," in Antennas and Propagation Society International Symposium, 2007 IEEE, June 2007, pp. 861-864.

[8] C. Sudhendra, A. Pillai, A. Madhu, and K. Rao, "A novel 6 to $14 \mathrm{ghz}$. thin radar absorber based on circular resistive patch fss," in Circuits, Controls and Communications (CCUBE), 2013 International conference on, Dec 2013, pp. 1-4.

[9] D. Xie, X. Liu, H. Guo, X. Yang, C. Liu, and L. Zhu, "Wideband absorber with multi-resonant griddedsquare fss for antenna rcs reduction," IEEE Antennas and Wireless Propagation Letters, vol. PP, no. 99, pp. 1-1, 2016.

[10] R. Dickie, R. Cahill, V. Fusco, H. Gamble, and N. Mitchell, "Thz frequency selective surface filters for earth observation remote sensing instruments," Terahertz Science and Technology, IEEE Transactions on, vol. 1, no. 2, pp. 450-461, Nov 2011.

[11] M. Bouslama, M. Traii, T. A. Denidni, and A. Gharsallah, "Beam-switching antenna with a new reconfigurable frequency selective surface," IEEE Antennas and Wireless Propagation Letters, vol. 15, pp. 1159-1162, 2016.

[12] A. Edalati and T. A. Denidni, "Frequency selective surfaces for beam-switching applications," IEEE Transactions on Antennas and Propagation, vol. 61, no. 1, pp. 195-200, Jan 2013.

[13] L. Kurra, M. P. Abegaonkar, A. Basu, and S. K. Koul, "Fss properties of a uniplanar ebg and its application in directivity enhancement of a microstrip antenna," IEEE Antennas and Wireless Propagation Letters, vol. 15, pp. 1606-1609, 2016.

[14] A. Pirhadi, H. Bahrami, and J. Nasri, "Wideband high directive aperture coupled microstrip antenna design by using a fss superstrate layer," IEEE Transactions on Antennas and Propagation, vol. 60, no. 4, pp. 2101-2106, April 2012. 
[15] D. Xie, X. Liu, H. Guo, X. Yang, C. Liu, and L. Zhu, "A wideband absorber with a multiresonant griddedsquare fss for antenna rcs reduction," IEEE Antennas and Wireless Propagation Letters, vol. 16, pp. 629632, 2017.

[16] J. P. Turpin, P. E. Sieber, and D. H. Werner, "Absorbing ground planes for reducing planar antenna radar cross-section based on frequency selective surfaces," IEEE Antennas and Wireless Propagation Letters, vol. 12, pp. 1456-1459, 2013.

[17] X. H.-Y., H. Zhang, K. Lu, and X.-f. Zeng, "A hollyleaf-shaped monopole antenna with low rcs for uwb application," Progress In Electromagnetics Research, vol. 117, pp. 35-50, 2011.

[18] S. Kitagawa, R. Suga, and O. Hashimoto, "Switchable reflector for radar cross section reduction of $\mathrm{x}$-band dipole array," in 2014 15th International Radar Symposium (IRS), June 2014, pp. 1-4.

[19] — - "Design of dipole antenna with reflector for rcs reduction using reradiation interference cancellation," in 2014 Asia-Pacific Microwave Conference, Nov 2014, pp. 1393-1395.

[20] S. Monni, G. Gerini, and A. Neto, "Frequency selective surfaces for the rcs reduction of low frequency antennas," in 2006 First European Conference on Antennas and Propagation, Nov 2006, pp. 1-6.

[21] N. Misran, R. Cahill, and V. Fusco, "Rcs reduction technique for reflectarray antennas," Electronics Letters, vol. 39, no. 23, pp. 1630-2-, Nov 2003.

[22] S. Genovesi, F. Costa, and A. Monorchio, "A wideband rcs reduction of slot array antennas," in $A n$ tennas and Propagation Society International Symposium (APSURSI), 2012 IEEE, July 2012, pp. 1-2.

[23] — , "Low-profile array with reduced radar cross section by using hybrid frequency selective surfaces," Antennas and Propagation, IEEE Transactions on, vol. 60, no. 5, pp. 2327-2335, May 2012.

[24] F. Costa and A. Monorchio, "Use of frequency selective surfaces for the reduction of radar cross section of antennas and scattering objects," in Prc. on 2nd ESA AntennaWorkshop on Antennas for Space Applications, October 2010.

[25] G. Machado, R. Cahill, and M. de Melo, "A low radar cross section dipole antenna array simulation," in Microwave and Optoelectronics Conference (IMOC), 2015 SBMO/IEEE MTT-S International, Nov 2015, pp. $1-5$.

[26] Y. Liu, Y. Hao, H. Wang, K. Li, and S. Gong, "Low rcs microstrip patch antenna using frequency-selective surface and microstrip resonator," IEEE Antennas and
Wireless Propagation Letters, vol. 14, pp. 1290-1293, 2015.

[27] (2016, Jan) United states frequency allocations. National Telecommunications and Information Administration. [Online]. Available: https://www.ntia.doc.gov/files/ntia/publications/2003allochrt.pdf

[28] B. Munk, Frequency Selective Surfaces: Theory and Design. Wiley, 2005. [Online]. Available: http://books.google.co.uk/books?id=9pNMhRQrpScC

[29] W. Li, C. Wang, Y. Zhang, and Y. Li, "A miniaturized frequency selective surface based on square loop aperture element," International Journal of Antennas and Propagation, vol. 2014, no. 701279, pp. 1-6, 2014.

[30] N. Marcuvitz and I. of Electrical Engineers, Waveguide Handbook, ser. Electromagnetics and Radar Series. P. Peregrinus, 1951. [Online]. Available: https://books.google.com.br/books?id=Ao34iFuNZgIC

[31] R. J. Langley and E. A. Parker, "Equivalent circuit model for arrays of square loops," Electronics Letters, vol. 18, no. 7, pp. 294-296, April 1982.

[32] A. d. S. Campos, Superfíes Seletivas em Frequência: Análise e projeto., IFRN, Ed. Natal: Editora IFRN, 2008.

[33] C. K. Lee and R. J. Langley, "Equivalent-circuit models for frequency-selective surfaces at oblique angles of incidence," IEE Proceedings H - Microwaves, Antennas and Propagation, vol. 132, no. 6, pp. 395-399, October 1985. 